
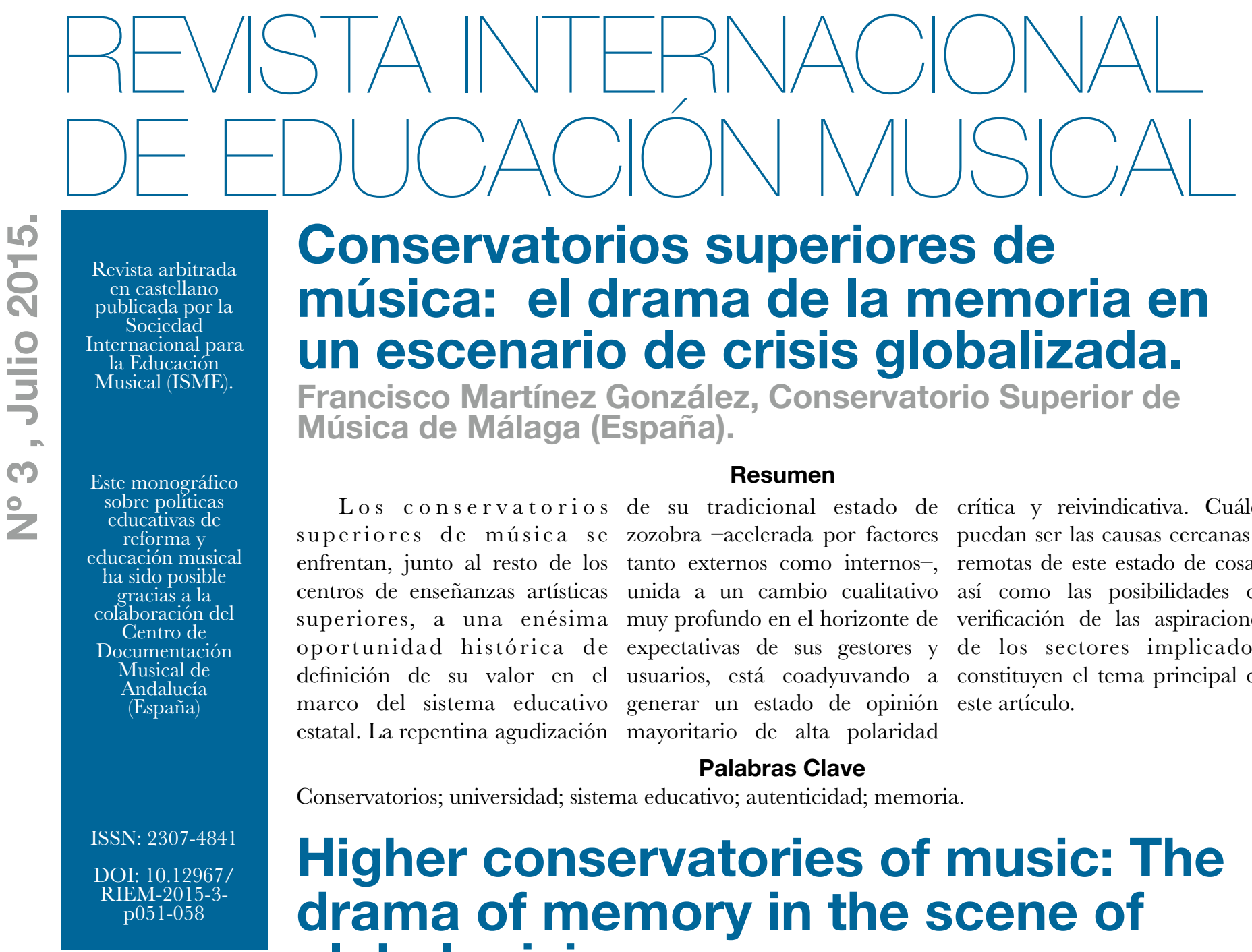

\title{
Conservatorios superiores de música: el drama de la memoria en un escenario de crisis globalizada.
}

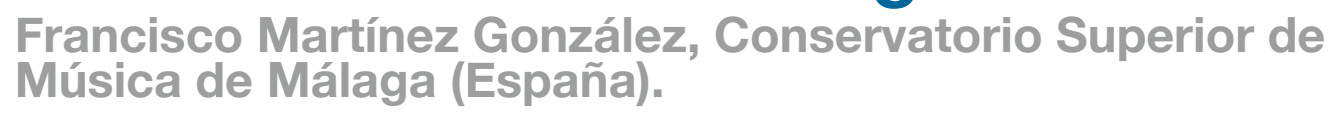
Música de Málaga (España).

\section{Resumen}

Los conservatorios de su tradicional estado de crítica y reivindicativa. Cuáles superiores de música se zozobra-acelerada por factores puedan ser las causas cercanas y enfrentan, junto al resto de los tanto externos como internos-, remotas de este estado de cosas, centros de enseñanzas artísticas unida a un cambio cualitativo así como las posibilidades de superiores, a una enésima muy profundo en el horizonte de verificación de las aspiraciones oportunidad histórica de expectativas de sus gestores y de los sectores implicados, definición de su valor en el usuarios, está coadyuvando a constituyen el tema principal de marco del sistema educativo generar un estado de opinión este artículo. estatal. La repentina agudización mayoritario de alta polaridad

\section{Palabras Clave}

Conservatorios; universidad; sistema educativo; autenticidad; memoria.

\section{Higher conservatories of music: The drama of memory in the scene of global crisis.}

\author{
Francisco Martínez González, Higher Conservatory of Málaga \\ (Spain).
}

\section{Abstract}

Higher Conservatories of intensification of their majority state of opinion with a Music confront for the traditional condition of highly critic and demanding thousandth time, alongside with precariousness - accelerated by polarity. The main subject of this the rest of higher artistic external as much as internal paper is to analyse near and education institutions, a new factors-, alongside with a very remote reasons of this state of historic opportunity of definition deep qualitative change about affairs, as well as the possibilities concerning their value in the the horizon of expectations of of realizing the aspirations of framework of the state education managers and users, is the involved sectors.5 system. The sudden collaborating to generate a

\section{Keywords}

Conservatories; University; national curriculum; authenticity; memory.

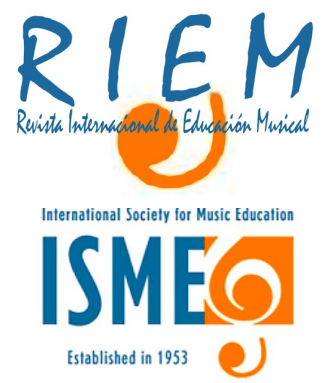


 drama de la memoria en un escenario de crisis globalizada.
}

\author{
por Francisco Martínez González, Conservatorio Superior de Música de Málaga (España).
}

\section{Autenticidad e inautenticidad}

La anómala situación de los conservatorios superiores de música en el conjunto del sistema educativo español lleva concitando desde hace más de medio siglo (por ceñirnos solo al período de tiempo de mayor intensidad crítica) debates y polémicas en relación con la naturaleza y la definición de estas instituciones, así como la de las enseñanzas que en ellas se ofrecen.

Para muchos de los que se desempeñan profesionalmente como docentes en estos centros, y desde luego para el que modestamente suscribe estas líneas, lo ininteligible de los conservatorios superiores estriba básicamente en un error de ubicación, en un problema, si se quiere, «geométrico»: los conservatorios no están donde deberían estar -la Universidad-, y de ahí que surja inevitablemente el problema de la disfunción, de la incongruencia entre lo que se debería ser y lo que se es, de la falta de concordancia entre lo que se nos exige alcanzar y los medios que se arbitran para lograrlo. De ahí también que su existencia se experimente como deficitaria, radicada en ese preciso e incesante malestar que irradia todo aquello que no ocupa su lugar correcto.

Esta situación y este sentir, junto con la visita reciente de algunos lugares de la filosofía de especial predilección, ha suscitado casi inevitablemente la resonancia de algunas palabras clave cuyo timbre querría compartir con los lectores. No se trata aquí en modo alguno de reflexión en un sentido sistemático, ni de emprender análisis que requerirían del analista otra vocación y otras dotes, sino de rozar siquiera el corolario existencial del problema, que también lo tiene, y no solo lógico o administrativo o legal.

Una de esas palabras clave, la primera, es la de «autenticidad», de hondo recorrido en la filosofía contemporánea. Para una inicial aproximación al concepto no se me ocurre mejor medio que el de apelar a la brillantez explicativa de José Ferrater Mora, quien lo define así en referencia a la que va a ser nuestra primera cata filosófica, José Ortega y Gasset:

Ortega y Gasset ha hablado con frecuencia de autenticidad e inautenticidad en el hombre como caracteres ontológicos de la realidad humana. En 1916 [...] describía un "yo auténtico" como la "base insobornable" de una vida humana; el "yo auténtico" es, en rigor, el "yo insobornable", esto es, el yo que, en el fondo y radicalmente, no puede dejar de ser lo que es.

Cuando el hombre llega a ser lo que es, entonces su vida es propia. El hombre cumple entonces con su vocación radical y con su "destino" (Ferrater, 1994a, Vol. I, p. 275).

Prima aquí, como vemos, el cumplimiento pindárico de «llegar a ser lo que se es»: solo entonces la vida es propia, dice Ortega, pues propiedad e impropiedad constituyen un par simétrico al de autenticidad e inautenticidad.

De Ortega, que por comunidad lingüística y cultural nos queda muy cerca, el paso obligado siguiente lleva ineludiblemente a una de las estrellas de la filosofía del siglo XX: Martin Heidegger. La agudeza de Heidegger no tiene parangón en el análisis de lo que George Steiner ha llamado el "sentir de la situación inauténtica del hombre en un ambiente de habla erosionada" (Steiner, 2001, p. 76). Digámoslo de nuevo con las palabras de Ferrater Mora: Heidegger ha hablado de autenticidad (Eigentlichkeit) e inautenticidad (Uneigentlichkeit) como modos de ser básicos del Dasein. [...] El Dasein puede, en efecto, "elegirse a sí mismo", es decir, "ganarse", en cuyo caso se apropia a sí mismo y se hace "auténtico". Puede también "no elegirse a sí mismo", es decir, "perderse", en cuyo caso deja de apropiarse a sí mismo y se hace "inauténtico" (no llega a ser lo que es) [...] (Ferrater, 1994 ${ }^{\text {b }}$, Vol. I, p. 275).

El Dasein (vale decir, el ser humano) de Heidegger es fundamentalmente posibilidad [Möglichkeit], y porque el Dasein es cada vez esencialmente su posibilidad, este ente puede en su ser «escogerse», ganarse a sí mismo, o bien puede «perderse», es decir, no ganarse jamás o solo ganarse aparentemente (Ferrater, 1994 ${ }^{\mathrm{b}}$ ).

$\mathrm{Y}$ es aquí, a continuación de Heidegger y de su concepción del Dasein como posibilidad, donde me gustaría introducir la tercera referencia filosófica: la de la malagueña María Zambrano. Para Zambrano la realidad está compuesta de distintos estratos o planos que discurren paralelos y que, de vez en cuando, se entrecruzan o se interfieren pudiendo llegar a desencadenar sucesos imprevistos. Esta concepción de la vida no es sino una forma de poetizar el mundo, al tiempo que exalta la trascendencia que cada acto, cada gesto, por nimio que pueda parecer, tiene en los distintos «planos morfológicos» de lo real. Se trata sin duda, de una creencia que desemboca en una actitud religiosa ante la vida. Nada es intrascendente y, como sostiene el misticismo sufí, el mundo inferior es el análogon del mundo de arriba. También Zambrano habla de esas cualidades que canalizan la acción hacia el espacio de la autenticidad o propiedad:

Ninguna acción verdadera trascendente se cumple sólo en uno de esos mundos o planos donde en verdad la vida humana se da. Y de ahí ha de venir la necesidad de la metáfora esencial. Si la piedra es sólo esta piedra que veo, si mi ver no la mira trasponiéndola en algo que está bajo ella, en algo que la soporta y la oprime, en algo que imprevisiblemente, en un movimiento ascensional, la hace templo, copa del cielo, el hombre, y aun quizá todo lo viviente, se queda sin lugar ${ }^{1}$ (Zambrano, 2004, p. 80).

El concepto central zambraniano de «acción verdadera» emerge en la cita anterior, dando a entender que toda aquella realización que realmente se desmarque de la heideggeriana «vida inauténtica» existe en una suerte de resonancia entre los distintos planos o cuerdas de la realidad. Como si eso que llamamos realidad fuese una suerte de impensable instrumento musical en el que la vibración por simpatía constituyera el símil correspondiente a esa genial intuición de los mundos o planos concomitantes, una idea que María Zambrano pudo leer espléndidamente desarrollada en la obra de su amigo, el gran escritor cubano José Lezama Lima.

Propiedad, autenticidad, acción verdadera: ¿qué tiene que ver todo esto con los conservatorios superiores de música?, ¿pueden proyectarse en el plano de la vida de las instituciones herramientas y conceptos en principio diseñados 
para el análisis de lo existencial humano? Y, de ser así, ¿en qué sentido las instituciones - los Conservatorios Superiores de Música, por ejemplo- pueden arrastrar una existencia «propia» o «impropia», «auténtica» o «inauténtica»? Antes de intentar dar respuesta a estas preguntas necesitamos introducir una instancia crítica más, a modo de corrección y de contraste: la memoria.

\section{La memoria como razón}

La memoria, Mnemósine, la madre de las Musas. Su relación con la música va más allá de las complejas redes tejidas por la mitología: constituye un vínculo del máximo poder de sugestión en el campo de la estética y, por ende, en el de la filosofía. Se trata de un motivo recurrente en los escritos de María Zambrano, cuya formulación más contundente aparece en las palabras que ella plasmó en un ensayo brillante, «La condenación aristotélica de los pitagóricos», de El hombre y lo divino:

Y el canto y la lira -armonía que es razón, pero también y siempre evocación- otra acción mágica, atraedora de almas, de recuerdos. La música es la diosa que sirve a la memoria. Es coherente también en este punto de leyenda de Pitágoras [sic] que le atribuye una prodigiosa, sobrehumana, memoria. La música nació para vencer el tiempo y la muerte, su seguidora (Zambrano, 1993, p. 84).

Un ensayo de más extensión y profundidad sobre este topos puede encontrarse en el capítulo IV de su Notas de un método. En ese lugar Zambrano escribe: "La música del pensamiento sobrepasa, pues que lo enhebra, el ir y venir de la memoria [...]" (Zambrano, 1989, p. 80). De una manera poética describe la autora ese traspasar, recoger, hilvanar la música -«la música del pensamiento»- los fragmentos dispersos de la memoria, dotando así de un sentido a lo vivido. Es lo epifánico de los momentos en que algo largamente incubado o padecido cobra significación y se ilumina en toda su trascendencia:

Todo lo que se abre dando a ver en unidad lo que ha aparecido como fragmentario y hasta inconexo y encontrado lo salva en rosa; en forma de rosa que admite innumerables pétalos, a partir de los cuatro iniciales [...] (Zambrano, 1989, p. 139).

En esta aproximación a la tríada Música-PensamientoMemoria se hace necesario realizar una mención a una última fuente filosófica: la representada por el alemán Theodor Wiesengrund Adorno. La ponderación de la importancia de la memoria en el pensamiento de este filósofo ha merecido consideraciones como esta de la profesora Marta Tafalla:

Esta categoría que apenas emerge en la superficie de sus escritos, mencionada escasa y brevemente, es la más oculta, pero desde los márgenes está centrando su pensamiento, justamente porque la filosofía debe alimentarse de lo marginado, de lo olvidado. Y quien nos lo retorna es la memoria (Tafalla, 2003, p. 195).

La memoria es medio para neutralizar la alienación, es vía para contrarrestar y corregir todo dominio, no importa cuál sea su origen, pues "si todo dominio consiste en el olvido de lo dominado, es la memoria la única que puede vencerlo" (Tafalla, 2003, p. 195-196). Con estas ideas acerca de la memoria, hay que reconocer a Adorno su contribución a la definición de lo que ha sido bautizado como razón anamnética, razón que razona y prescribe, proyecta y siente desde una alerta constante por rescatar del olvido.

Recuerda Marta Tafalla que fue Johann Baptist Metz quien puso en circulación la expresión «razón anamnética» en un famoso artículo así titulado, "Anamnetische Vernunft" (1989). Allí ponía en evidencia Metz la doble crisis del pensamiento europeo - crisis de las ciencias del espíritu y crisis del cristianismo-, señalando al mismo tiempo la falla interna que recorre a este último. En efecto, el cristianismo bebe de una doble fuente: por un lado el núcleo milenario del judaísmo, por otro la sistemática racional de ascendencia griega. Esa doble dependencia genera tensiones y disonancias, pero puede ser trascendida o corregida mediante el redescubrimiento de un contenido racional propio del judaísmo y diferente del helénico: la razón anamnética y la memoria. Metz sintetizó esa orientación particular de la racionalidad judía con el aforismo Denken als Andenken, als geschichtliches Eingedenken [Pensar como recordar, como memoria histórica] (Tafalla, 2003). Según Metz, fue "la primera generación de la Escuela de Frankfurt, en especial Benjamin y Adorno, así como Rosenzweig, Levinas, Bloch y Jonas, [...] los filósofos que han recuperado la tradición judía de la memoria para incorporarla a la filosofía occidental" (ibíd., p. 204).

¿Es esta razón anamnética, en tanto que determinada por el «ir y venir de la memoria», una razón musical? ¿No constituye la música una suerte de cauce para el ejercicio refinado de la memoria, de la ligazón entre aconteceres puros, en el sentido de no lastrados de referencialidad, como son los que se suceden durante el curso de la audición estética? ¿Nos hemos olvidado de la memoria? Pues la memoria, y la educación basada en la interiorización mnemónica, han sido durante siglos elemento vertebrador y modulador de la sensibilidad (Steiner, 2001), ¿qué consecuencias (epistemológicas, éticas, políticas) tiene su marginación? Y en la línea de las consideraciones que nos ocupan: ¿están nuestras instituciones dedicadas a la enseñanza musical de grado superior también en trance de perder enteramente su memoria?, ¿podría ser esta, la memoria, una posibilidad o un argumento para garantizar un atisbo de autenticidad?, ¿ejercerá la memoria sus derechos como instancia crítica para regular la reflexión acerca de lo que esas instituciones son o deberían aspirar a ser?

\section{Música y universidad: ¿un binomio improbable?}

Las consideraciones anteriores encuentran ahora su espacio de aplicación y su norma. Creo firmemente que gran parte del problema que afecta a los conservatorios -gran parte también de las pasiones, el malestar, la frustración y hasta el resentimiento que esa situación puede llegar a generar- tiene que ver con un olvido de un segmento sustancial de nuestra historia. Es necesario hacer un ejercicio de memoria, un esfuerzo de recuperación que pueda traernos de nuevo ante los ojos el todo o una parte de ese material genético originario.

La música ha sido parte de la enseñanza universitaria durante más tiempo que el que lleva durando su exilio de ese mismo territorio, aunque su encuadre en el marco de la universidad española no haya sido unívoco a lo largo de un período tan largo. Como escribe el profesor Antonio Martín Moreno, catedrático de Musicología de la Universidad de Granada:

La presencia de la música en la universidad española ha pasado por distintas etapas y situaciones desde la Antigüedad hasta nuestros días. [...] En la actualidad, la música reduce su presencia a varias titulaciones, siendo la 
más novedosa la de Historia y Ciencias de la Música. El futuro más inmediato pasará por aunar fuerzas entre los distintos estamentos y el acercamiento paulatino entre esta institución docente y aquellas que imparten otros estudios artísticos, unas dedicadas a la pedagogía y la historiografía, y las otras a la interpretación (Martín Moreno, 2005).

La bibliografía sobre la presencia de la música en la universidad es considerable, aunque está todavía por escribirse una monografía definitiva sobre la historia de la enseñanza musical universitaria (ibíd.). La narración del por todos lados lamentable divorcio entre la música (en sus diferentes facetas, tales como la historiográfica, la analítica, la creativa, la interpretativa) y el mundo universitario puede presenciarse en el instante de su consumación simbólica en relación con una de las universidades más señeras del acervo académico hispánico, la de Salamanca. Así, nos recuerda Martín Moreno (2005, pp. 63.64):

Dámaso García Fraile (2004) ha corregido los datos hasta ahora difundidos sobre la supresión de la cátedra de Música de Salamanca, que no tuvo lugar en la reforma de 1807 del Ministro Caballero [...], sino unos cuantos años antes, en 1792, fecha en que Carlos IV aprobó el plan propuesto por la Universidad para la creación de un nuevo Colegio de Filosofía. En la Provisión Real en la que se dispone la supresión de la Cátedra de Música se reitera que la enseñanza de la música en la Universidad de Salamanca consta de dos partes: la música práctica, que seguirá practicándose en la orquesta de la Universidad creada en 1738 y la música teórica de la que se responsabiliza a los catedráticos de Matemáticas, por lo que la supresión de la dotación no supondría "ningún perjuicio a la enseñanza, puesto que lo científico de aquel arte debe y puede dejarse a los catedráticos de Matemáticas" [...].

Un año más tarde, en 1793, falleció el último catedrático de Música de la Universidad salmantina, Juan Antonio Aragüés, cuyo sustituto desde 1778 fue Manuel José Doyagüe que no llegó a ser Catedrático de Música porque, como afirma García Fraile (2004), dicha Cátedra fue suprimida antes de fallecer el maestro Aragüés que la ocupaba. Es cierto que esta supresión adquiere carácter definitivo en la reforma educativa de 1807 del ministro Caballero en la que desaparece cualquier referencia a las cátedras y a la enseñanza universitaria de la música.

Durante más de cinco siglos la presencia de la música en la Universidad española, en su aspecto teórico, investigador, especulativo y práctico, fue una realidad viva y dinámica, ofreciendo al acervo cultural occidental la aportación de los Ramos de Pareja, o Francisco Salinas en el siglo XVI, por citar sólo a los catedráticos más universalmente conocidos. Esta relación entre teoría y práctica musical es manifiestamente declarada por Francisco Salinas: "Y yo aprendí que también a la música se puede aplicar lo que Vitrubio dice con tanta razón de la arquitectura, a saber, que los que intentan ejercitarse sólo manualmente, sin estudiar, no pueden realizar nada eficazmente, y los que confían tan sólo en la teoría y en las letras, dan la impresión de que persiguen la sombra, no la realidad. Por fin, los que aprenden una y otra cosa, como adornados por todas las armas, llegan a conseguir pronto una gran autoridad en lo que se propusieron".

La mención, al final de la cita anterior, de las palabras de Francisco Salinas es providencial. Durante mucho tiempo se ha estado utilizando la apelación al carácter práctico de las enseñanzas musicales, específicamente en su faceta relativa a la especialidad de Interpretación, como un argumento contra su estatus intelectual, contra su posible equiparación a otras enseñanzas consideradas más elevadas; pero, como dice Salinas, solo "los que aprenden una y otra cosa [la teoría de la música y la práctica interpretativa], como adornados por todas las armas, llegan a conseguir pronto una gran autoridad en lo que se propusieron".

Habla Salinas de «conseguir autoridad». Pues bien, de eso mismo, de autoridad, de conquista de la legitimidad y el derecho -el que prestan solo los procesos democráticamente planteados de expresión de voluntades colectivas- queremos hablar a continuación.

\section{Breve recorrido histórico-legislativo en lo que se refiere a las enseñanzas artísticas superiores: 1970-2013.}

A la vista de los recursos presentados por las Universidades de Granada ${ }^{2}$, Málaga ${ }^{3}$, Sevilla ${ }^{4}$ y Politécnica de Valencia ${ }^{5}$, las recientes sentencias del Tribunal Supremo que anulan varios artículos del Real Decreto 1614/2009, de 26 de octubre, por el que se establece la ordenación de las Enseñanzas Artísticas Superiores reguladas en la LOE han supuesto una auténtica convulsión en los centros (Conservatorios Superiores de Música, de Danza, Escuelas Superiores de Arte Dramático, etc.) en los que se imparten esos estudios.

¿Por qué la música, en toda su diversidad de planteamientos y aproximaciones, no es disciplina universitaria? ¿Por qué las sentencias del Supremo invocadas arriba han representado el (amargo) despertar de un sueño, pero también una enésima posibilidad de reabrir el debate sobre el destino de los centros superiores de enseñanzas artísticas? Para encontrar respuestas a estas y otras preguntas, debemos empezar recordando que ya la Ley 14/1970, de 4 de agosto, General de Educación prescribía que los estudios de Bellas Artes, Música y Arte Dramático se integraran en la Universidad, a través de su famosa (y lamentablemente irresuelta) Disposición transitoria segunda:

Las Escuelas Superiores de Bellas Artes, los Conservatorios de Música y las Escuelas de Arte Dramático se incorporarán a la Educación universitaria en sus tres ciclos en la forma y con los requisitos que reglamentariamente se establezcan (p. 12543).

Desde entonces ha llovido mucho, se han sucedido diversas tentativas de acercamiento al mundo universitario, se ha enriquecido el argumentario de los que defienden tal acercamiento y, más importante aún, se ha aumentado la base social de reivindicación y protesta por la situación de indefinición administrativa en la que se encuentran los centros de enseñanzas artísticas superiores. Aunque un relato exhaustivo de todo lo acontecido no cabe en estas páginas, sí queremos ofrecer a continuación una selección muy apretada de algunos hechos importantes ${ }^{6}$ :

- En 1978 las escuelas superiores de Bellas Artes se transformaron en facultades universitarias mediante un Real Decreto, mientras que las enseñanzas superiores de música y arte dramático no lo hicieron.

- Se crea, en 1988, el Comité para la Incorporación de la Música a la Universidad, que reunió más de 4.000 firmas y la adhesión de importantes instituciones -entre ellas los conservatorios de Madrid y Valencia, la Orquesta Nacional de España y la de la Radiotelevisión Española, entre otras- y numerosas personalidades del mundo de la música y la cultura en general: Teresa Berganza, Alfredo Krauss, Camilo José Cela, Luis de Pablo, Jesús López Cobos, José Carreras, y Alicia de Larrocha, entre otras muchas. 
- En 1997, Antonio Embid Irujo - a la sazón catedrático de Derecho Administrativo de la Universidad de Zaragoza- recomienda mediante un informe, encargado por la Asociación Española de Centros Superiores de Enseñanzas Artísticas (ACESEA), integrar las Enseñanzas Artísticas Superiores (EEAASS) en el sistema universitario como una de las vías más plausibles para la regularización de estos centros.

- En 2005, ACESEA - ante la Comisión de Educación del Congreso, para informar acerca del proyecto de Ley Orgánica de Educación en lo referente a las EEAASS, y cuando las posiciones de los dos grandes partidos no eran opuestas a la integración de las EEAASS en la Universidad-, logra imponer su opinión de dejar fuera del sistema universitario a los mencionados estudios.

- En 2006, se aprueba la Ley Orgánica 2/2006, de 3 de mayo, de Educación, que establece las denominadas enseñanzas artísticas superiores, que agrupan los estudios superiores de música y danza, las enseñanzas de arte dramático, las enseñanzas de conservación y restauración de bienes culturales y los estudios superiores de artes plásticas y diseño (Artículo 58.3). Asimismo, y siguiendo el legislador de nuevo la recomendación de ACESEA, deja a las EEAASS fuera del sistema universitario, aunque no se renuncia a la futura transformación universitaria de las mismas.

- En 2009, concretamente el 14 de abril, la Coordinadora de Enseñanzas Artísticas Superiores (CEEAASSSS) hace público el manifiesto Por la plena integración de las EEAASS en la Universidad, que recaba más de 7.000 firmas.

Ese mismo año, el 27 de octubre se publica en el BOE el Real Decreto 1614/2009, de 26 de octubre, por el que se establece la ordenación de las enseñanzas artísticas superiores reguladas por la Ley Orgánica 2/2006, de 3 de mayo, de Educación, que, a diferencia de esta última, denomina a los títulos de EEAASS de Grado, en perfecta identidad nominal con los títulos universitarios.

- En 2011, tres Conservatorios Superiores de Música de Andalucía y uno de Danza -el único de toda la Comunidad Autónoma- aprueban en sus claustros el manifiesto Por unas EEAASS de calidad en Andalucía, el cual recoge entre sus reclamaciones "la integración de los nueve centros que imparten enseñanzas artísticas superiores en Andalucía en las respectivas universidades de las ciudades en las que desarrollan su labor" (p. XXX). En febrero de 2012 se sumaría el claustro del Conservatorio Superior de Música de Jaén.

El 22 de noviembre de ese mismo año, el Consejo de Gobierno de la Universidad de Sevilla, en colaboración con el Conservatorio Manuel Castillo de Sevilla y la Universidad Frederic Chopin de Varsovia, aprueba la Memoria de verificación de un nuevo Título Oficial de Máster Universitario en Enseñanzas Artísticas de Música, pionero en el ámbito de las enseñanzas artísticas de música en la comunidad autónoma andaluza y en España.

- En 2012, concretamente del 13 al 16 de enero, se dictan las sentencias de la Sala de lo ContenciosoAdministrativo, sección cuarta, del Tribunal Supremo, anulando, entre otros artículos, el número 8 del Real Decreto 1614/2009, que otorgaba la denominación de Graduado o Graduada a los titulados de las EEAASS.
El 14 de febrero, los directores de los cuatro Centros de EEAASS en Galicia -apoyándose en la aprobación de un manifiesto que pide "la integración plena y definitiva de las enseñanzas artísticas superiores en la institución universitaria" en los claustros y consejos escolares de los Conservatorios Superiores de Música de Vigo y La Coruña, Escuela de Restauración de Pontevedra y Escuela Superior de Arte Dramático de $\mathrm{Vigo}^{-}$, piden integrarse en la Universidad.

El 17 de febrero de ese año, el claustro del Conservatorio Superior de Música de Jaén aprueba el manifiesto Por unas EEAASS de calidad en Andalucía.

El 23 de febrero, el sindicato Comisiones Obreras (CCOO), a través de la Federación de Enseñanza, publica una nota de prensa en la que propone "abrir el debate y estudiar la incorporación de las EEAASS al sistema universitario, considerando la diversidad de sectores implicados, la necesidad de apostar por ritmos y modelos flexibles acordes a cada contexto sectorial y territorial y a la protección de los derechos laborales del personal que actualmente está ejerciendo su labor docente en estas enseñanzas"7.

El 5 de marzo, el claustro del Real Conservatorio Superior de Música de Madrid, en sesión extraordinaria, aprueba un manifiesto que pide "la urgente necesidad de que se den los pasos necesarios para incorporar las enseñanzas de nuestro Centro en el espacio universitario pertinente, [...] dotando definitivamente de rango universitario a nuestro Centro y sus enseñanzas mediante cualquier forma admitida en derecho" .

El 10 de marzo se constituye en el Real Conservatorio Superior de Música de Madrid la Plataforma por la integración de las EEAASS en el sistema universitario, inspirada en un manifiesto que lleva el mismo nombre y que ha sido aprobado ya por once centros.

El 20 de marzo, el rector de la Universidad de Granada, en el acto de inauguración de la Feria de Arte Contemporáneo de la Facultad de Bellas Artes, dice en su discurso: "[...] la Universidad de Granada está «abierta» a acoger a las enseñanzas artísticas que actualmente están fuera"9 (p. 57).

- En 2013, por último, varios son los acontecimientos importantes que jalonan el último año considerado en esta relación cronológica. Uno de los más significativos fue la celebración, durante los días 26 y 27 de abril, del encuentro La Universidad y los Conservatorios Superiores de Música, auspiciado por el Rectorado de la Universidad de Granada, el Departamento de Historia y Ciencias de la Música de la UGR, los cinco Conservatorios Superiores de Música de Andalucía, la Universidad Internacional de Andalucía y la Universidad de Oviedo. En el texto de justificación del encuentro podía leerse: "Se pretende que representantes de las Universidades (en su doble aspecto institucional y académico), directores de los Conservatorios Superiores de Música, así como los responsables de la política educativa, expongan sus reflexiones en un momento en el que se está debatiendo un nuevo marco legislativo para la Educación. En definitiva, se trata de exponer criterios, intercambiar puntos de vista y proponer soluciones al ya viejo problema de la normalización de las enseñanzas musicales en España, en el marco del Espacio Europeo de Educación Superior"10. El encuentro, todo un 
éxito desde el punto de vista organizativo, contó también con presencia institucional: en él intervinieron D. ${ }^{a}$ María Teresa Varón García, Directora General de Evaluación y Ordenación Educativa de la Consejería de Educación de la Junta de Andalucía, y D. ${ }^{\text {a Violeta }}$ M $^{\text {a }}$ Baena Gallé, Jefa del Servicio de Ordenación de Enseñanzas Artísticas y Deportivas.

Asimismo, la Ley de Mejora de la Calidad Educativa (LOMCE), aprobada en el pleno del Congreso el 10 de octubre de 2013, incluye en su articulado este importante recordatorio: "Las Administraciones educativas podrán adscribir centros de Enseñanzas Artísticas Superiores mediante convenio a las Universidades, según lo indicado en el artículo 11 de la Ley orgánica 6/2001, de 21 de diciembre, de Universidades" (p. 97893).

Por último, ADICOAN (Asociación de Directores de Conservatorios Elementales y Profesionales de Música de Andalucía) ha aprobado en su última asamblea, celebrada el 25 de octubre de 2013, la adhesión a los manifiestos que piden la adscripción de los conservatorios superiores de música a las correspondientes universidades, así como la integración plena y definitiva de las enseñanzas artísticas superiores en el sistema universitario.

Hace muchos años hablaba José María Cagigal, artífice de la conversión de los INEF en facultades universitarias, de "las siempre equívocas 'equivalencias"” (en Chinchilla y otros, 2008, p. 5). Más de treinta años después de aquellas palabras del gran humanista vasco, los conservatorios superiores de música y los centros de enseñanzas artísticas en general parecen estar en la tesitura de no querer seguir soportando por más tiempo esa dolorosa situación de equívoco e indefinición.

\section{A modo de conclusión}

Los conservatorios superiores de música necesitan resolver de una vez por todas el problema de la falta de un estatuto propio claro y definido: como una suerte de entes híbridos, a medio camino entre una estructura administrativa que los mantiene ligados a las enseñanzas secundarias y una advocación funcional que los llama a desempeñar una función insustituible en el espacio de la superior, su fallida ubicación geométrica los aboca a una situación de perpetua crisis esquizoide que se hace cada vez más insostenible. Necesitan los conservatorios salir del limbo de suspensión topológica en el que vienen consumiéndose desde hace décadas, y esa salida, que hemos llamado también búsqueda de su autenticidad, está siendo reclamada cada vez con más contundencia. Para un número exponencialmente creciente de usuarios, la universidad es la caja de resonancia natural de las enseñanzas artísticas en general y de las musicales en particular. Dicho en términos técnicos, para las EEAASS la Universidad es el único a priori que permitiría el acontecer de su verdad, entendiendo esta en el sentido de una existencia propia o auténtica.

La ubicación de las enseñanzas musicales en el espacio universitario generaría de suyo un enfoque diferente de la enseñanza de la música en los estadios iniciales de la educación, promoviendo una regularización y dignificación de la misma en esos otros niveles.

Además, sería irresponsable no atender al cambio cualitativo operado en los claustros de los conservatorios, en los que se está verificando la titulación de un número creciente de doctores, lo cual allana, sin duda, el camino hacia la adscripción y la posterior integración universitaria.

El problema, que en grandísima medida es de voluntad política, reclama también de los que lo padecen la lucha contra la desidia, el derrotismo y la amnesia. Solo la memoria -el esfuerzo de entronque retrospectivo- puede realizar la apertura del horizonte futuro. Es una obligación ética la de recordar nuestro origen y no desaprovechar esta nueva ocasión histórica de afirmación y cambio.

\section{Notas}

${ }^{1}$ La cursiva es nuestra.

${ }^{2}$ Recurso contencioso-administrativo 122/2009, fecha de sentencia de 13/01/2012.

${ }^{3}$ Recurso 123/2009, sentencia de 16/01/2012.

${ }^{4}$ Recurso 124/2009, sentencia de 16/01/2012.

${ }^{5}$ Recurso 127/2009, sentencia de 16/01/2012.

${ }^{6}$ Agrademos a ADEMAS (Asociación para la Defensa de las Enseñanzas Musicales y Artísticas) el habernos facilitado muchos de los datos que se exponen en este artículo. Una relación más detallada de los acontecimientos aquí recogidos puede consultarse en nuestro artículo (Martínez González, 2014).

${ }^{7}$ http://www.fe.ccoo.es/ensenanza/

Sistema_Educativo:Ensenanzas_Artisticas:Inicio:317111-CG.OO._pide_una_solucion_urgente_a_la_problematica_de _los_titulos_de_las_ensenanzas_artisticas_superiores

${ }^{8}$ Manifiesto del Claustro de Profesores del Real

Conservatorio Superior de Música de Madrid. Disponible en http://www.beckmesser.com/manifiesto-del-claustro-deprofesores-del-real-conservatorio-superior-de-musica-demadrid/.

${ }^{9}$ http://canalugr.es/medios-impresos/item/download/ 45862.

${ }^{10}$ http://secretariageneral.ugr.es/pages/tablon/*/noticiascanal-ugr/el-rector-inaugura-el-encuentro-la-universidad-ylos-conservatorios-superiores-de-musica-en-la-ugr.

\section{Referencias citadas}

Chinchilla, J. L., López Fernández, L., Romero, Ó. y Romero, S. (2008). La integración de los Institutos Nacionales de Educación Física en la Universidad (1961-1992). Revista de la Facultad de CC de la Educación, Universidad de Sevilla, 8, 1-13.

Ferrater, J. (1994ª). Diccionario de filosofía, vol. I. Voz «Autenticidad, auténtico». Barcelona: Ariel.

Ferrater, J. (1994b). Diccionario de filosofia, vol. I. Voz « Dasein». Barcelona: Ariel.

Jefatura del Estado (1970). Ley 14/1970, de 4 de agosto, General de Educación y Financiamiento de la Reforma Educativa. Boletín Oficial del Estado, 187, 12525-12546. Disponible en http://www.boe.es/diario_boe/txt.php? id=BOE-A $-1970-852$.

Jefatura del Estado (2006). Ley Orgánica 2/2006, de 3 de mayo, de Educación. Boletín Oficial del Estado, 106, 1-107. Disponible en http://www.boe.es/buscar/act.php? id=BOE-A-2006-7899.

Jefatura del Estado (2013). Ley Orgánica 8/2013, de 9 de diciembre, para la mejora de la calidad educativa. Boletín Oficial del Estado, 295, 97858-97921. Disponible en http:// www.boe.es/diario_boe/txt.php?id=BOE-A-2013-12886. 
Martín Moreno, A. (2005). Pasado, presente y futuro de la Musicología en la universidad española. Revista Interuniversitaria de Formación del Profesorado, 19(1), 53-76.

Martínez González, F. (2014). Los conservatorios superiores de música: querencias universitarias en términos de voluntad, reivindicación y proyecto. MAR, Música de Andalucía en la Red, 4, 28-36. Disponible en http:// mar.ugr.es/static/MAR_Revista/*/4/articulos/losconservatorios-superiores-de-musica-en-andaluciaquerencias-universitarias-en-terminos-de-voluntadreivindicacion-y-proyecto.

Metz, J. B. (1989). Anamnetische Vernunft. Anmerkungen eines Theologen zur Krise der Geisteswissenschaften. En

A. Honneth, T. McCarthy, C. Offe, y A. Wellmer (Ed.). Zwischenbetrachtungen. Im Prozeß der Aufklärung. Frankfurt: Suhrkamp.

Steiner, G. (2001). Sobre la dificultad y otros ensayos. México: Fondo de Cultura Económica.

Tafalla, M. (2003). Theodor W. Adorno. Una filosofia de la memoria. Barcelona: Herder.

Zambrano, M. (1989). Notas de un método. Madrid: Mondadori.

Zambrano, M. (1993). El hombre y lo divino. México: Fondo de Cultura Económica.

Zambrano, M. (2004). De la aurora. Madrid: Tabla Rasa. 


\section{Sobre el Autor}

\section{Francisco Martínez González}

Ha realizado el conjunto de su carrera musical en el Conservatorio Superior de Málaga.

En 1988 gana por oposición una cátedra de Repentización, Transporte y Acompañamiento, En 1990, tras superar las pruebas de selección correspondientes, obtiene la cátedra de Historia y Estética de la Música. Es licenciado en Geografia e Historia, especialidad Historia del Arte (Musicología) por la Universidad de Granada.

$\mathrm{Ha}$ formado parte del equipo de investigadores encargado de elaborar el Catálogo del Archivo de Música de la Catedral de Málaga, y en su actividad como musicólogo e investigador se inscribe su continua participación en ciclos de conferencias, congresos, simposios y colaboraciones en revistas especializadas. En 2005 fue invitado a dictar una conferencia sobre La modernidad de Falla en el Trinity College de Londres.

Ha sido responsable de una columna de crítica musical en el diario La Opinión de Málaga. En 2008 defendió su tesis doctoral en la Universidad de Granada. El libro Los músicos del 27, del que es coordinador, vio la luz en 2010, en una edición conjunta de la Universidad de Granada y el Centro de Documentación Musical de Andalucía. Actualmente es responsable artístico del Ciclo de música de cámara de la Orquesta Filarmónica de Málaga.

Desde julio de 2012 es director del Conservatorio Superior de Música de Málaga.

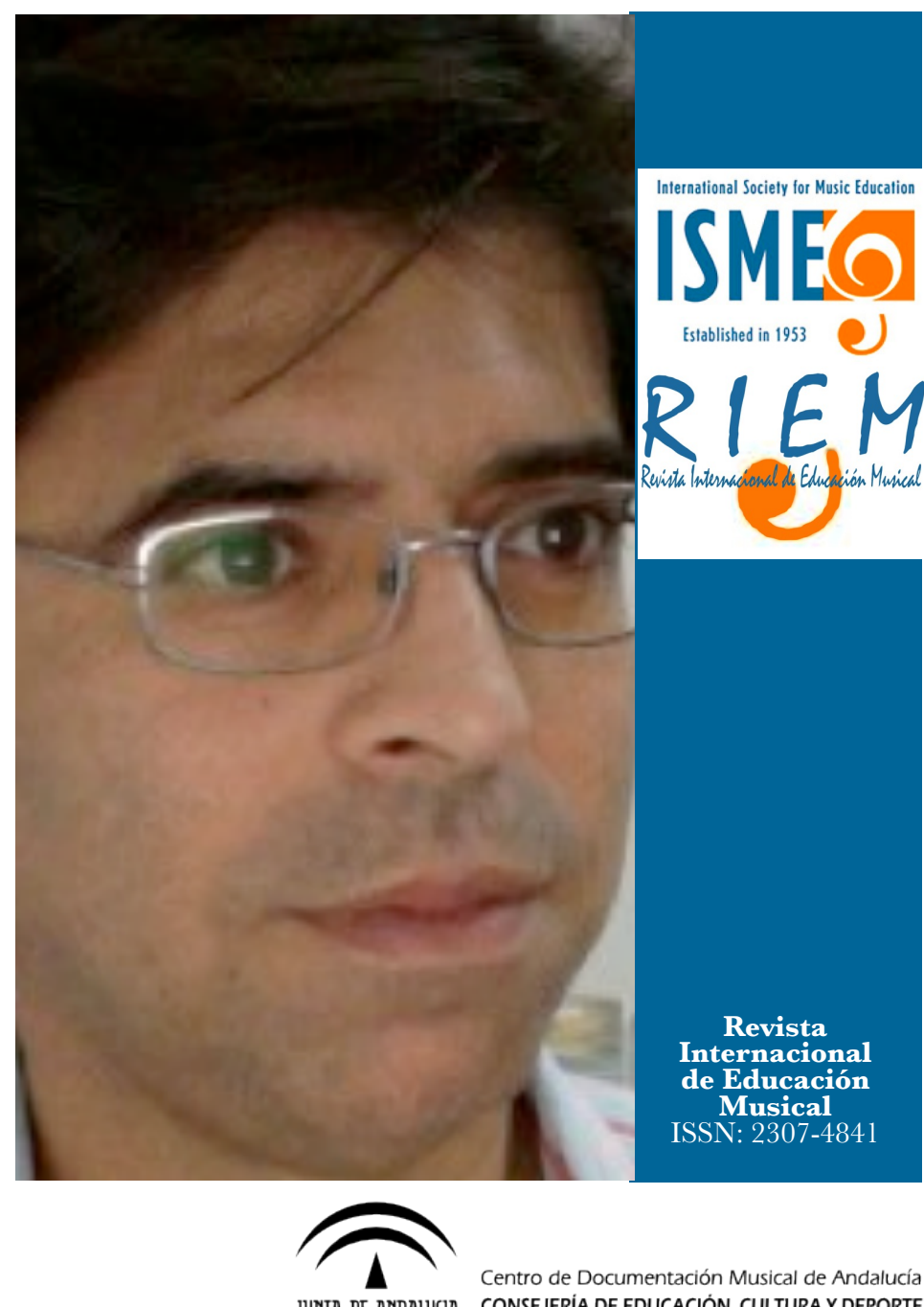

\section{Francisco Martínez González}

Conservatorio Superior de Música de Málaga

Pza. Maestro Artola, 2

JUNTA DE AMDALUCIA CONSEJERIA DE EDUCACIÓN, CULTURA Y DEPORTE

29013 Málaga. España.

f.m.gonzalez.edu@juntadeandalucia.es

\section{EQUIPO EDITORIAL}

Editor:

José Luis Aróstegui Plaza, Universidad de Granada (España)

\section{Editora Adjunta:}

Rosa María Serrano Pastor, Universidad de Zaragoza (España)

\section{Consejo Editorial}

\section{Carlos Abril, Universidad de Miami (Estados Unidos)}

María del Carmen Aguilar, Instituto Coral de Buenos Aires (Argentina)

Miquel Alsina Tarrés, Universidad de Gerona (España)

Carmen Carrillo Aguilera. Universidad Internacional de Cataluña (España)

Sergio Luiz Figueiredo, Universidad del Estado de Santa Catarina (Brasil)

Patricia Adelaida González, Universidad Autónoma de Chihuahua (México)

Claudia Gluschankof, Instituto Levinsky (Israel)

Josep Gustems Carnicer, Universidad de Barcelona (España)

María Cecilia Jorquera Jaramillo, Universidad de Sevilla (España)

Gotzon Ibarretxe Txakartegi, Universidad del País Vasco (España)

Ana Laucirica Larrinaga, Universidad Pública de Navarra (España)

Ana Lucia Louro, Universidad Federal de Santa María (Brasil)
Isabel Cecilia Martínez, Universidad Nacional de La Plata (Argentina)

Teresa Mateiro, Universidad del Estado de Santa Catarina (Brasil)

María Teresa Moreno, Universidad Laval (Canadá)

Graça Boal Palheiros, Instituto de Educación de Oporto (Portugal)

Jèssica Pérez Moreno, Universidad Autónoma de Barcelona (España) y Universidad de Londres (Reino Unido)

Gabriel Enrique Rusinek Milner, Universidad Complutense de Madrid (España)

Patrick K. Schmidt, Universidad de Ontario Occidental (Canadá)

Favio Shifres, Universidad Nacional de La Plata (Argentina)

Maria dels Àngels Subirats Bayego, Universidad de Barcelona (España)

António Ângelo Ferreira Vasconcelos, Instituto de Educación de Setúbal (Portugal)

Maria Helena Vieira, Universidad del Miño (Portugal)

Gloria Patricia Zapata Restrepo, Universidad de Antioquía Colombia) 